\title{
Bootstrap tests of multiple inequality restrictions on variance ratios
}

\author{
Jeff Fleming $^{\text {a }}$, Chris Kirby ${ }^{\text {b,* }}$, Barbara Ostdiek ${ }^{\text {a }}$ \\ a Jones Graduate School, Rice University, Houston, TX 77005, United States \\ b John E. Walker Department of Economics, Clemson University, Box 341309, Clemson, SC 29634-1309, United States
}

Received 9 February 2005; received in revised form 1 December 2005; accepted 8 December 2005

Available online 19 April 2006

\begin{abstract}
We develop a block bootstrap method for testing multiple inequality restrictions on variance ratios. The proposed test has reasonable size and power in the presence of strong persistence in conditional variances, making it well suited to applications in financial econometrics.
\end{abstract}

(C) 2005 Elsevier B.V. All rights reserved.

Keywords: Composite hypothesis; Stationary bootstrap; Least favorable configuration

JEL classification: $\mathrm{C} 12 ; \mathrm{C} 15$

\section{Introduction}

Variance ratio tests have many uses in economics and finance. One well-known application is testing the random-walk model of stock prices (Lo and MacKinlay, 1988). Under the random-walk model, the variance of $k$-period stock returns divided by the variance of single-period stock returns equals the horizon $k$. Although asymptotic methods can be used to test this restriction, studies of related tests suggest that these methods are unreliable if the data display strong persistence in conditional variances (see, e.g., Andersen et al., 2001). Resampling and subsampling methods, on the other hand, appear to perform well under such circumstances, at least in the context of testing the random-walk model (see, e.g., Whang and Kim, 2003).

\footnotetext{
* Corresponding author. Tel.: +1 864656 0553; fax: +1 8646564192.

E-mail address: cmkirby@clemson.edu (C. Kirby).
} 
In other applications, the analysis of variance ratios generates multiple inequality restrictions. Fleming et al. (in press), for example, study the ratio of trading-period to nontrading-period return variances for weather-sensitive commodities. Under their model, this ratio should be smaller during the weather-sensitive season than during the rest of the year. Even if the analysis does not focus on variance ratio inequalities, it may suggest hypotheses that can be formulated in terms of these inequalities. For instance, Engle and Colacito (in press) use portfolio variances to assess the relative performance of competing estimators of the conditional covariance matrix of asset returns. The hypothesis that a given covariance matrix estimator is superior to all others considered can be expressed as a set of variance ratio inequalities.

In this paper, we propose a bootstrap test of multiple inequality restrictions on variance ratios. Since inequality restrictions do not specify unique parameter values, they are more challenging to test than equality restrictions. The usual strategy for resolving the ambiguity inherent in the null hypothesis is to construct critical values using the parameter configuration least favorable to the alternative hypothesis (see, e.g., Perlman, 1969; Wolak, 1987, 1989). There are a few studies, such as White (2000), that use bootstrap methods to implement inequality tests. However, little is known about the performance of these tests under autoregressive conditional heteroscedasticity (ARCH), especially for restrictions that involve unconditional variances. We use simulations to address this issue.

\section{Test statistic and bootstrap procedure}

Suppose that $Y_{t}=\left(Y_{0 t}, Y_{1 t}, \ldots, Y_{n t}\right)^{\prime}$ is generated by a stationary stochastic process of the form

$$
Y_{t}=\mu+\varepsilon_{t},
$$

where $\varepsilon_{t}$ is vector white noise. ${ }^{1}$ Let $\mathrm{VR}_{i}=\sigma_{0}^{2} / \sigma_{i}^{2}$ denote the ratio of $\operatorname{var}\left(Y_{0 t}\right)$ to $\operatorname{var}\left(Y_{i t}\right)$. To illustrate our methodology, we consider the null and alternative hypotheses

$$
\mathrm{H}_{0}: \mathrm{VR}_{i} \leq 1 \forall i=1,2, \ldots, n ; \quad \mathrm{H}_{\mathrm{A}}: \mathrm{VR}_{i}>1 \text { for some } i \in(1,2, \ldots, n) .
$$

Other types of inequality restrictions can be tested using the same approach. For example, Fleming et al. (in press) test hypotheses about differences in variance ratios.

Let $Y=\left(Y_{1}, Y_{2}, \ldots, Y_{T}\right), \quad \mu_{T}(Y)=(1 / T) \sum_{t=1}^{T} Y_{t}$, and $e_{t}=Y_{t}-\mu_{T}(Y)$. Our test of $\mathrm{H}_{0}$ is based on the statistic

$$
\theta_{T}(Y)=\max _{1 \leq i \leq n} \sqrt{T}\left(\frac{\mathrm{VR}_{i T}(Y)-1}{\gamma_{i T}(Y)}\right),
$$

where

$$
\operatorname{VR}_{i T}(Y)=\frac{\sigma_{0 T}^{2}(Y)}{\sigma_{i T}^{2}(Y)},
$$

\footnotetext{
${ }^{1}$ We specify a serially uncorrelated process for ease of exposition. The results generalize to autoregressive specifications in a straightforward fashion.
} 


$$
\begin{aligned}
\sigma_{0 T}^{2}(Y) & =(1 / T) \sum_{t=1}^{T} e_{0 t}^{2}, \quad \sigma_{i T}^{2}(Y)=(1 / T) \sum_{t=1}^{T} e_{i t}^{2}, \text { and } \gamma_{i T}(Y) \text { denotes a consistent estimator of } \\
\gamma_{i} & =\sigma_{i}^{-2}\left(\sum_{s=-\infty}^{\infty} E\left(\left(\varepsilon_{0 t}^{2}-\mathrm{VR}_{i} \varepsilon_{i t}^{2}\right)\left(\varepsilon_{0 t-s}^{2}-\mathrm{VR}_{i} \varepsilon_{i t-s}^{2}\right)\right)\right)^{1 / 2} .
\end{aligned}
$$

It is straightforward to show that $\sqrt{T}\left(\mathrm{VR}_{i T}(Y)-\mathrm{VR}_{i}\right) / \gamma_{i T}(Y) \stackrel{d}{\rightarrow} N(0,1){ }^{2}$ Thus, $\theta_{T}(Y)$ is simply the largest of the $t$-statistics obtained by setting $\mathrm{VR}_{i}=1$ for each $i=1,2, \ldots, n$. We studentize the variance ratios because using asymptotically pivotal or nearly pivotal statistics improves the performance of bootstrap methods.

Now suppose we have $T$ observations $y=\left(y_{1}, y_{2}, \ldots, y_{T}\right)$ from the process in Eq. (1) and let $F_{T}(c)=P\left(\theta_{T}(Y) \leq c\right)$ denote the probability that $\theta_{T}(Y)$ is less than or equal to $c$. We use a block bootstrap approach to approximate $F_{T}(c)$. First, we construct a resample $y^{*}=\left(y_{1}^{*}, y_{2}^{*}, \ldots, y_{T}^{*}\right)$ using the stationary bootstrap of Politis and Romano (1994). ${ }^{3}$ The resample is such that, in general, if $y_{i}^{*}=y_{t}$, then $y_{i+1}^{*}=y_{t+1}$ with probability $p$ and $y_{i+1}^{*}$ is drawn randomly from $\left(y_{1}, y_{2}, \ldots, y_{T}\right)$ with probability $1-p$. This delivers an expected block length of $\bar{L}=1 /(1-p)$. Second, we calculate

$$
\theta_{T}^{*(1)}=\max _{1 \leq i \leq n} \sqrt{T}\left(\frac{\left.\mathrm{VR}_{i T}(Y)\right|_{Y=y^{*}}-\left.\mathrm{VR}_{i T}(Y)\right|_{Y=y}}{\left.\gamma_{i T}(Y)\right|_{Y=y^{*}}}\right) .
$$

Finally, after replicating the first two steps $M$ times to obtain $\theta_{T}^{*(1)}, \theta_{T}^{*(2)}, \ldots, \theta_{T}^{*(M)}$, we approximate $F_{T}(c)$ by

$$
\hat{F}_{T}(c)=\frac{1}{M} \sum_{m=1}^{M} I\left(\theta_{T}^{*(m)}-\left.\theta_{T}(Y)\right|_{Y=y} \leq c\right),
$$

where $I(\cdot)$ denotes the indicator function. This corresponds to bootstrapping the distribution of $\theta_{T}(Y)$ under the least favorable configuration (LFC), i.e., when all the inequalities are binding (Wolak, 1987). ${ }^{4}$ The resulting critical value for testing $\mathrm{H}_{0}$ at significance level $v$ is given by $\inf \left\{c: \hat{F}_{T}(c) \geq 1-v\right\}$.

\section{Monte Carlo experiments}

We use simulations to investigate the size and power of the test under various assumptions about the data generating process. For our baseline case we take $Y_{t} \mid y_{t-1}, \ldots, y_{1}$ to be $N(0, \Sigma)$ with $\Sigma_{i j}=\sigma_{i} \sigma_{j} \rho_{i j}$. We set $n=5, \sigma_{0}=1$, and $\rho_{i j}=0: 3$ for all $i \neq j$. Since the test size depends on the number of binding inequalities, we consider three configurations: $\mathrm{VR}_{i}=1$ for (i) $i=1$; (ii) $i \leq 3$; and (iii) $\forall i$. The non-binding inequalities have $\mathrm{VR}_{i}=0.5$. For the power calculations, we set $\mathrm{VR}_{1}=1.5$ and keep all the other

\footnotetext{
${ }^{2}$ To see this, consider an exactly identified GMM system based on the moments $E\left(\varepsilon_{0 t}\right)=0, E\left(\varepsilon_{i t}\right)=0$, and $E\left(\varepsilon_{0 t}^{2}-\mathrm{VR}_{i} \varepsilon_{i t}^{2}\right)=0$. We assume that $E\left(\varepsilon_{i t}^{2} \varepsilon_{j t}\right)=0$ for all $i=0,1, \ldots, n$ and $j=0,1, \ldots, n$, so that $\sqrt{T}\left(\operatorname{VR}_{i T}(Y)-\operatorname{VR}_{i}\right)$ is asymptotically independent of $\sqrt{T}\left(\mu_{0 T}(Y)-\mu_{0}\right)$ and $\sqrt{T}\left(\mu_{i T}(Y)-\mu_{i}\right)$. This can easily be relaxed if skewness is important.

${ }^{3}$ See Goncalves and de Jong (2003) for a proof of the first-order asymptotic validity of the stationary bootstrap under the existence of only slightly more than second moments.

${ }^{4}$ The LFC implies that $\sqrt{T}\left(\operatorname{VR}_{i T}(Y)-1\right)$ is asymptotically distributed as $N\left(0, \gamma_{i}^{2}\right) \forall i=1,2, \ldots, n$. Since the bootstrap distribution of $\sqrt{T}\left(\left.\mathrm{VR}_{i T}(Y)\right|_{Y=y^{*}}-\left.\mathrm{VR}_{i T}(Y)\right|_{Y=y}\right)$ is centered at zero, it follows that the bootstrap delivers critical values for the LFC. See White (2000).
} 
Table 1

Monte Carlo evidence on size and power

\begin{tabular}{|c|c|c|c|c|c|c|c|c|c|}
\hline \multirow[t]{2}{*}{ Observations } & \multirow[t]{2}{*}{ Binding } & \multicolumn{4}{|c|}{ Homoscedastic data } & \multicolumn{4}{|c|}{$\underline{\text { GARCH data }}$} \\
\hline & & $\bar{L}=5$ & $\bar{L}=10$ & $\bar{L}=20$ & $\bar{L}=40$ & $\bar{L}=5$ & $\bar{L}=10$ & $\bar{L}=20$ & $\bar{L}=40$ \\
\hline \multicolumn{10}{|c|}{ Panel A: Estimated size of $\theta_{T}$} \\
\hline \multirow[t]{3}{*}{$T=320$} & 1 & 0.013 & 0.011 & 0.013 & 0.016 & 0.070 & 0.036 & 0.027 & 0.018 \\
\hline & 3 & 0.036 & 0.031 & 0.035 & 0.051 & 0.157 & 0.087 & 0.061 & 0.058 \\
\hline & 5 & 0.059 & 0.049 & 0.054 & 0.072 & 0.216 & 0.128 & 0.095 & 0.083 \\
\hline \multirow[t]{3}{*}{$T=640$} & 1 & 0.012 & 0.011 & 0.016 & 0.019 & 0.063 & 0.044 & 0.032 & 0.022 \\
\hline & 3 & 0.035 & 0.032 & 0.038 & 0.045 & 0.153 & 0.096 & 0.068 & 0.052 \\
\hline & 5 & 0.054 & 0.058 & 0.055 & 0.066 & 0.216 & 0.141 & 0.095 & 0.078 \\
\hline \multirow[t]{3}{*}{$T=1280$} & 1 & 0.010 & 0.017 & 0.012 & 0.016 & 0.063 & 0.044 & 0.026 & 0.024 \\
\hline & 3 & 0.029 & 0.034 & 0.037 & 0.040 & 0.154 & 0.098 & 0.074 & 0.056 \\
\hline & 5 & 0.047 & 0.046 & 0.055 & 0.059 & 0.213 & 0.138 & 0.104 & 0.080 \\
\hline \multirow[t]{3}{*}{$T=2560$} & 1 & 0.016 & 0.014 & 0.017 & 0.019 & 0.068 & 0.047 & 0.032 & 0.024 \\
\hline & 3 & 0.035 & 0.032 & 0.038 & 0.035 & 0.160 & 0.100 & 0.074 & 0.049 \\
\hline & 5 & 0.057 & 0.049 & 0.060 & 0.053 & 0.226 & 0.145 & 0.110 & 0.070 \\
\hline \multicolumn{10}{|c|}{ Panel B: Estimated power of $\theta_{T}$} \\
\hline \multirow[t]{3}{*}{$T=320$} & 0 & 0.928 & 0.919 & 0.881 & 0.817 & 0.654 & 0.567 & 0.421 & 0.297 \\
\hline & 2 & 0.927 & 0.919 & 0.881 & 0.817 & 0.664 & 0.572 & 0.431 & 0.313 \\
\hline & 4 & 0.927 & 0.919 & 0.881 & 0.818 & 0.673 & 0.579 & 0.444 & 0.322 \\
\hline \multirow[t]{3}{*}{$T=640$} & 0 & 0.999 & 0.999 & 0.998 & 0.996 & 0.875 & 0.809 & 0.710 & 0.604 \\
\hline & 2 & 0.999 & 0.999 & 0.998 & 0.996 & 0.876 & 0.811 & 0.711 & 0.606 \\
\hline & 4 & 0.999 & 0.999 & 0.998 & 0.996 & 0.879 & 0.811 & 0.711 & 0.609 \\
\hline \multirow[t]{3}{*}{$T=1280$} & 0 & 1.000 & 1.000 & 1.000 & 1.000 & 0.985 & 0.971 & 0.948 & 0.908 \\
\hline & 2 & 1.000 & 1.000 & 1.000 & 1.000 & 0.985 & 0.971 & 0.948 & 0.908 \\
\hline & 4 & 1.000 & 1.000 & 1.000 & 1.000 & 0.985 & 0.971 & 0.949 & 0.908 \\
\hline \multirow[t]{3}{*}{$T=2560$} & 0 & 1.000 & 1.000 & 1.000 & 1.000 & 1.000 & 1.000 & 0.999 & 0.999 \\
\hline & 2 & 1.000 & 1.000 & 1.000 & 1.000 & 1.000 & 1.000 & 0.999 & 0.999 \\
\hline & 4 & 1.000 & 1.000 & 1.000 & 1.000 & 1.000 & 1.000 & 0.999 & 0.999 \\
\hline
\end{tabular}

The table documents the size and power of the test under homoscedasticity and under ARCH. Panel A reports the estimated size at $5 \%$. Panel B reports the estimated power at $5 \%$ based on a violation of the first inequality. "Binding" is the number of binding inequalities. The parameter settings are given in the text. All results are based on 2000 simulation trials with 2000 bootstrap replications per trial.

parameters the same. To illustrate the impact of ARCH on the test, we take $Y_{t} \mid y_{t-1}, \ldots, y_{1}$ to be $N\left(0, H_{t}\right)$ with $H_{i j t}=h_{i t}{ }^{1 / 2} h_{j t}{ }^{1 / 2} \rho_{i j}$, where $h_{i t}$ and $h_{j t}$ are univariate $\operatorname{GARCH}(1,1)$ processes of the form

$$
h_{i t}=\zeta_{i}+\beta_{i} h_{i t-1}+\alpha_{i} y_{i t-1}^{2},
$$

with $\zeta_{i}=\sigma_{i}^{2}\left(1-\alpha_{i}-\beta_{i}\right)$. We set $\alpha_{i}=0.05, \beta_{i}=0.9$ and $h_{i 0}=\sigma_{i}^{2}$ for all $i=0,1, \ldots, n$. The values of $\rho_{i j}$ are identical to the baseline case.

We generate samples of 320, 640, 1280, and 2560 observations from each process. To construct $\theta_{T}(Y)$, we need to estimate $\gamma_{i}$. This is accomplished using a standard kernel-based estimator of the form

$$
\gamma_{i T}(Y)=\sigma_{i T}^{-2}(Y)\left(\omega_{i T}^{(0)}(Y)+\sum_{s=1}^{q} 2\left(1-s(1+q)^{-1}\right) \omega_{i T}^{(s)}(Y)\right)^{1 / 2},
$$


where

$$
\omega_{i T}^{(s)}(Y)=\frac{1}{T} \sum_{t=s+1}^{T}\left(e_{0 t}^{2}-\mathrm{VR}_{i T}(Y) e_{i t}^{2}\right)\left(e_{0 t-s}^{2}-\mathrm{VR}_{i T}(Y) e_{i t-s}^{2}\right) .
$$

The lag truncation parameter $q$ is set $40 \%$ larger than the expected block length, i.e., $q=1.4 \bar{L}$. We consider four values of $\bar{L}: 5,10,20$, and 40. In all cases, we perform 2000 simulation trials with $M=2000$ bootstrap replications per trial.

Table 1 summarizes the results. Panel A reports the estimated size of the test at a nominal significance level of $5 \%$. The rejection rate under homoscedasticity is not sensitive to either the value of $T$ or the value of $\bar{L}$. The rate is close to $5 \%$ for the scenario in which all of the inequalities are binding, and falls (as expected) as the number of binding inequalities decreases. This relation follows from using the LFC to construct critical values. In contrast, the rejection rate under $\mathrm{ARCH}$ is sensitive to the value of $\bar{L}$. For the scenario in which all of the inequalities are binding, the rejection rate falls from about $22 \%$ with $\bar{L}=5 \%$ to $7 \%$ or $8 \%$ with $\bar{L}=40$. Thus, using a moderate to large block length seems prudent if ARCH is suspected.

Panel B reports the estimated power of the test at a nominal significance level of $5 \%$ based on a violation of the first inequality restriction. We focus on the results for $\bar{L}=40$, i.e., the expected block length that shows the least evidence of size distortions. In general, the test has good power for the homoscedastic case. The rejection rate exceeds $99 \%$ for $T \geq 640$. Not surprisingly, the presence of $\mathrm{ARCH}$ leads to a reduction in power, but even in this case the rejection rate exceeds $90 \%$ for $T \geq 1280$. To put this in context, five years of daily stock return data is about 1260 observations, and samples larger than this are common place in the volatility modeling literature. Therefore, it appears that the power under $\mathrm{ARCH}$ is adequate for empirically relevant values of $T$.

\section{Concluding remarks}

We develop a bootstrap test of multiple inequality restrictions on variance ratios. Our test is easy to implement and has reasonable size and power for data that display strong persistence in conditional variances provided the choice of block length is not too conservative. Given these findings, it would be interesting to investigate the performance of our test using an automatic block-length selection procedure like that of Politis and White (2004). We leave this to future research.

\section{References}

Andersen, T., Bollerslev, T., Das, A., 2001. Variance-ratio statistics and high-frequency data. Journal of Finance 56, $305-327$.

Engle, R., Colacito, R., in press. Testing and valuing dynamic correlations for asset allocation. Journal of Business and Economic Statistics.

Fleming, J., Kirby, C., Ostdiek, B., in press. Information, trading, and volatility: evidence from weather-sensitive markets. Journal of Finance.

Goncalves, S., de Jong, R., 2003. Consistency of the stationary bootstrap under weak moment conditions. Economics Letters $81,273-278$.

Lo, A., MacKinlay, C., 1988. Stock market prices do not follow random walks: evidence from a simple specification test. Review of Financial Studies 1, 41-66. 
Perlman, M., 1969. One-sided testing problems in multivariate analysis. The Annals of Mathematical Statistics 40, $549-567$.

Politis, D., Romano, J., 1994. The stationary bootstrap. Journal of the American Statistical Association 89, 1303-1313.

Politis, D., White, H., 2004. Automatic block-length selection for the dependent bootstrap. Econometric Reviews 23, 53-70.

Whang, Y., Kim, J., 2003. A multiple variance ratio test using subsampling. Economics Letters 79, 225-230.

White, H., 2000. A reality check for data snooping. Econometrica 68, 1097-1126.

Wolak, F., 1987. An exact test for multiple inequality and equality constraints in the linear regression model. Journal of the American Statistical Association 82, 782-793.

Wolak, F., 1989. Testing inequality constraints in linear econometric models. Journal of Econometrics 41, 205-235. 